\title{
Assessment of Agaricus bisporus Mushroom as Protective Agent Against Ultraviolet Exposure
}

\author{
Chae Yeon Hwang ${ }^{1}$, Yuniwaty Halim ${ }^{2}$, Marcelia Sugata ${ }^{3}$, Dela Rosa ${ }^{4}$, Sherlyn Putri Wijaya ${ }^{5}$ \\ and Eden Steven ${ }^{1,5}$
}

\begin{abstract}
Mushrooms are versatile materials with applications including but not limited to food, cosmetics, and pharmaceutical industries. In this work, the potential of the common button mushroom, Agaricus bisporus, as a protective agent against ultraviolet exposure was assessed. The assessment was done by investigating the radical scavenging activity, sun protecting capability, and tyrosinase inhibiting properties of Agaricus bisporus ethanolic extract. The extraction was carried out using absolute ethanol as its solvent at low to room temperatures. The bioactive components of the ethanolic extract were analysed for its phenolic and flavonoid contents quantitatively, while other phytochemical agents were analysed qualitatively. The Agaricus bisporus ethanolic extract was found exhibit varying degree of activity in all of the assessment. We found low radical scavenging ability with \%RSA IC50 of $\sim 5456 \mu \mathrm{g} / \mathrm{mL}$, low to moderate sun protecting factor of $\sim 5.355$ at $5000 \mathrm{ppm}$ concentration, and high tyrosinase inhibition property with IC50 of $\sim 2 \mu \mathrm{g} / \mathrm{mL}$. The high tyrosinase inhibition property was found to correlate with relatively high total phenolic content of $\sim 1143 \mathrm{mg} \mathrm{GAE} / 100 \mathrm{~g}$ for Agaricus bisporus and the presence of terpenoid in the ethanolicextract.

${ }^{*}$ Corresponding author
\end{abstract}

\section{Keywords}

Mushroom, UV filter, tyrosinase inhibition, sun protecting factor, DPPH assay, phythochemical screening

\section{Introduction}

Research of NASA scientists have evidenced that ground ultraviolet radiation (UVR) reaching the Earth's surface has increased significantly over the past few decades ${ }^{1}$. UVR lies on the left of visible light in the electromagnetic spectrum, meaning its wavelengths are relatively shorter and they contain more energy. These short wavelengths of UVR enable UVR to penetrate the human skin ${ }^{2}$. Moderate exposure to UVR is actually required by humans to promote conversion of the 7-dehydrocholesterol into provitamin D and then into its functional form of vitamin D. However, excessive amounts of UVR has been proven to induce negative effects on human health as it may trigger overproduction of the reactive oxygen species (ROS) and cause DNA damage. This damage then cause adverse effects, such as abnormal transformation of skin cells and immunosuppression towards malignant cells. The typical phenotypic changes vary from benign changes such as wrinkles to fatal conditions such as blindness and the development of tumours and/or cancerous cells ${ }^{12}$.

In response to UV radiation along with its DNA-damaging effects, the human skin employs endogenous mechanisms to prevent and/or cure the radiative effects. Examples of the mechanisms include apoptosis of mutated cells, overexpression of antioxidant genes, rapid repair of DNA, thickening of the epidermis and, most importantly, increased melanogenesis ${ }^{34}$. Melanogenesis, a biosynthetic pathway responsible for the production of melanin in the epidermal layer ${ }^{5}$, is vital as the melanin produced as a result of the process, serves as a UVR absorbent, antioxidant and ROS scavenger ${ }^{3}$. Thus increased melanogenesis is essential to photoprotection against the chronic effects of UV radiation including photo-aging, photodamage and photo-carcinogenesis, otherwise known as skin cancer. Especially post-exposure to UVR, melanin begins developing toxic properties. In-vitro studies testify to melanin's ability to react and alter DNA negatively. Pheomelanin, additionally, has been evidenced to be especially vulnerable to photodegradation, generating hydrogen peroxide, superoxide anions, causing mutations in melanocytes and inducing apoptosis of cells among other dangers of increased melanin ${ }^{6}$. Overaccumulation of melanin is also unfavourable as it might lead to a myriad of disorders, including, hyperpigmentation, neurodegeneration and even skin cancer ${ }^{7}$. Tight regulation of skin melanin levels is thus crucial since both abnormally high and low melanin levels pose a potential danger for human well-being.

\footnotetext{
${ }^{1}$ Applied Science Academy, Sekolah Pelita Harapan Lippo Village, Tangerang, Indonesia

${ }^{2}$ Department of Food Technology, Universitas Pelita Harapan, Tangerang Indonesia

${ }^{3}$ Department of Biology, Universitas Pelita Harapan, Tangerang, Indonesia ${ }^{4}$ Department of Pharmacy, Universitas Pelita Harapan, Tangerang, Indonesia

${ }^{5}$ Emmerich Research Center, Jakarta, Indonesia
}

\section{Corresponding author:}

Eden Steven, Applied Science Academy, Sekolah Pelita Harapan Lippo Village, Tangerang, Indonesia; Emmerich Research Center, Jakarta, Indonesia 
Regulation of melanin levels can be achieved indirectly through adjustments of tyrosinase activity. Tyrosinase is a rate-limiting enzyme responsible for the first step of melanin synthesis - conversion of tyrosine into L- 3,4dihydroxyphenylalanine (DOPA) ${ }^{57}$. The other steps in melanogenesis are mostly spontaneous, therefore they require no catalyst. Due to these factors, overproduction of melanin is almost always due to overexpression of tyrosinase ${ }^{58}$. The search for tyrosinase inhibitors is thus of utmost importance and has been an attractive aspect of research for the cosmetics and food industry as well as a central endeavour for the medical industry in inhibiting and minimizing the damaging effects of UVR.

Amongst the candidates for tyrosinase inhibitors are mushrooms. Mushrooms have been long known to be rich in bioactive agents ${ }^{9}$, such as phenolic compounds. Additionally, mushrooms are appreciated for their antioxidant activity, primarily free radical scavenging effects, leading them to be valuable natural sources for pharmaceutical and nutritional purposes. Previous research, namely $\mathrm{Ga}_{c}$ secka et al. ${ }^{7}$ 's investigates the abundant composition of bioactive compounds and phenolic acids in seven strains of the Agaricus genus of mushrooms, one of which include the Agaricus bisporus white capped mushroom, evidencing the antioxidant and antiradical activities of this genus of mushrooms. While the sources from Chang et al. ${ }^{10}$ and Boonsong et al. ${ }^{2}$, do not study the specific Agaricus genus of mushrooms, they too evidence the value of mushrooms in antioxidant capabilities (mainly free radical scavenging, phenolic and flavonoid).

On melanin-reducing capabilities, the work of Zaidi et al. ${ }^{4}$, having recognized a lack of research augmenting the claim that mushroom extracts can be effective as melanogenic agents, have investigated the "effect of purified mushroom tyrosinase of Agaricus bisporus on B16F10 melanocytes for the melanin production via blocking pigment cell machinery" and thus have testified that the mushroom Agaricus bisporus is in fact a viable agent for melanogenesis inhibition.

Agaricus bisporus, commonly known as the white button mushroom, are amongst the most conventional, cultivated gourmet species of mushrooms. It is one of the most popular edible mushrooms, native to European and North American grasslands. The mushroom belongs to the Agaricus genus of the Agaricacae family. The mushroom is recognized for its hemispherical white cap and stem, which flattens out through maturity, and is the young origin of the Portobello mushroom. A. bisporus contains various known beneficial nutrients such as vitamin $\mathrm{D}$, potassium and sodium. Its consumption is also associated with a decreased risk of breast cancer and prevention of oxidative stress associated diseases $^{211}$.

Despite prior works evidencing the potential for Agaricus bisporus to be natural sources of antioxidants and tyrosinase inhibitors, minimal work has been done to assess the potential of Agaricus bisporus as a preventive and curative agent against ultraviolet exposure. Thus in this work, we aim to correlate and understand the role of the secondary metabolites of Agaricus bisporus ethanolic extracts with regards with its antioxidant, sun-protecting-factor (SPF), and anti-tyrosinase inhibiting properties. The identification of secondary metabolites is done quantitatively with regards to total phenolic and total flavonoid contents, while other phytochemical screening is done qualitatively.

\section{Materials and Methods}

\section{Sample Preparation}

Cultivated gourmet mushroom species Agaricus bisporus is first collected and chopped into fine pieces. Then the finely chopped whole Agaricus bisporus was freeze dried for 72 hours. Freeze dried sample was ground using dry spice grinder into fine powder form.

\section{Ethanol Extraction}

$25 \mathrm{~g}$ of freeze-dried mushroom powder was extracted with $250 \mathrm{~g}$ of ethanol absolute. The maceration was left to sit for 24 hours at RT. The supernatant was filtered through Whatman filter paper in a vacuum pump. The recovered supernatants, after the filter process, were combined and the ethanol was removed by rotary evaporation. The obtained extract was stored in an amber sample vial, at $4{ }^{\circ} \mathrm{C}$.

\section{DPPH Radical Scavenging Assay}

The DPPH assay aims to investigate the free radical scavenging activity of antioxidants (AH) towards the purplecoloured DPPH in et-OH, conducted as described by Lamien-Meda et al. methodology ${ }^{12}$. Samples were prepared by serial-dilution method to yield 2, 4, 6, 8, and $10 \mathrm{ppm}$ concentrations. Antioxidant assay is performed by adding $1 \mathrm{~mL}$ of $0.2 \mathrm{mM}$ DPPH and $0.8 \mathrm{~mL}$ of ethanol (et-OH). All the samples were prepared in triplicate, vortexed for $1 \mathrm{~min}$ and incubated in the dark for $30 \mathrm{~min}$ at room temperature. The absorbance of each sample and control was measured against $2 \mathrm{~mL}$ of ethanolic DPPH as blank on UV-Vis Spectrophotometer (Hitachi U1800) at wavelength of $517 \mathrm{~nm}$. Percentage of DPPH inhibition was calculated using the formula (Equation 1), similar to the methodology used by Kumara et al. ${ }^{13}$. The radical scavenging activity percentage (\%RSA) can be calculated from Equation 1.

$$
\% R S A=\frac{A_{\text {Control }}-A_{\text {Sample }}}{A_{\text {Control }}} * 100 \quad \text { Eq. } 1 .
$$

where $\mathrm{A}_{\text {Control }}$ is the absorbance of $0.2 \mathrm{mM}$ ethanolic DPPH only and $\mathrm{A}_{\text {Sample }}$ is the absorbance of the reacting mixture, with the addition of the mushroom sample. IC50 value (concentration at 50\% inhibition) was obtained from the linear regression of the data points and extracting the concentration at $50 \%$ inhibition value.

\section{Sun Protecting Factor (SPF) Test}

Methodology used is as described in Romulo et al.'s ${ }^{14}$. The absorption characteristics of the sunscreen agents against UVB (290-320 nm) can be determined in vitro by utilizing UV spectrophotometry with the following Equation 2 below:

$$
S P F=C F * \sum_{290}^{320} E E(\lambda) * I(\lambda) * A b s(\lambda) \quad \text { Eq. } 2 .
$$

where: EE (l) - erythemal effect spectrum; I (1) - solar intensity spectrum; Abs (1)- absorbance of sunscreen product ; $\mathrm{CF}$ - correction factor $(=10)$. The values of EE $\mathrm{x} \mathrm{I}$ at different wavelengths are given in Table 1 according to work done by Romulo et al's. ${ }^{14}$. 
Table 1. EExI (normalized) at each wavelength. ${ }^{14}$

\begin{tabular}{ll}
\hline Wavelength (nm) & EE x I (normalized) \\
290 & 0.0150 \\
295 & 0.0817 \\
300 & 0.2874 \\
305 & 0.3278 \\
310 & 0.1864 \\
315 & 0.0839 \\
320 & 0.0180 \\
Total & 1 \\
\hline
\end{tabular}

Sample was dissolved in ethanol to a concentration of 5,000 ppm. The absorbance of the sample solution was then read at wavelengths 290 - $400 \mathrm{~nm}$ with $5 \mathrm{~nm}$ interval. Ethanol was used as the blank, negative control. To obtain the SPF value, the absorbances of the sample at various wavelengths listed in Table 1 are obtained and then plugged into Equation 2. Their summation gives the total SPF value.

\section{Tyrosinase Inhibition}

Tyrosinase inhibition activity was determined according to the modified method of Chan et al, ${ }^{15}$ or Burger et al. ${ }^{16}$. Sample was dissolved in dimethyl sulfoxide (DMSO) to a concentration of $10,000 \mathrm{ppm}$ and then diluted in potassium phosphate buffer ( $50 \mathrm{mM}, \mathrm{pH} 6.8)$ to $0.05 \mathrm{ppm}$. All steps were conducted at room temperature. Samples with different concentration $(70 \mu \mathrm{l})$ was incubated with $30 \mu \mathrm{l}$ of the tyrosinase enzyme (333 U/ml in phosphate buffer, $\mathrm{pH}$ 6.8) for $5 \mathrm{~min}$, followed by the addition of $2 \mathrm{mM}$ of the substrate, L-tyrosine $(110 \mu \mathrm{l})$ and further incubated for 30 min at RT, ensuring that effect of light is limited. Kojic acid was used as positive control. The mixture of sample and other components, except L-tyrosine, was used as a blank. Absorbance reading was performed at $475 \mathrm{~nm}$. The percentage of tyrosinase inhibition was calculated using Equation 3:

$$
\% \text { inhibition }=\frac{A_{\text {Control }}-A_{\text {Sample }}}{A_{\text {Control }}} * 100
$$

where, \%inhibition is the tyrosinase inhibition percentage, $\mathrm{A}_{\text {control }}$ is the absorbance of the control solution and $\mathrm{A}_{\text {sample }}$ is the absorbance of the sample solution. For each sample concentration, the absorbance was recorded in triplicate manner.

\section{Total Phenolic Content by Folin Reagant Method}

Total phenolic content in A. bisporus extracts was determined using the Folin-Ciocalteu reagent using the method of Javanmardi et al. ${ }^{17}$. Serial dilution of gallic acid stock solution was made with the concentration of $100 \mathrm{ppm}$, 80 ppm, 60 ppm, 40 ppm and 20 ppm. For Folin-Ciocalteu stock solution, $10 \mathrm{~mL}$ of Folin reagent was diluted into 100 $\mathrm{mL}(10 \% \mathrm{w} / \mathrm{v})$ of distilled water and then stored away from light. $\mathrm{Na}_{2} \mathrm{CO}_{3}$ (Sodium Carbonate) solution was prepared by diluting $7.5 \%(\mathrm{w} / \mathrm{v})$ of $\mathrm{Na}_{2} \mathrm{CO}_{3}$ in distilled water solvent. Standard curve was prepared by combining $1.5 \mathrm{~mL}$ of the Folin-Ciocalteu reagent and $0.3 \mathrm{~mL}$ of gallic acid into a test tube. The mixture was vortexed and incubated in the dark for 5 minutes at room temperature. After incubation, $1.2 \mathrm{~mL}$ of $\mathrm{Na}_{2} \mathrm{CO}_{3}$ was added and vortexed once more. The solutions were then incubated in the dark for 15 minutes. The absorbance of each sample was measured against 0.3 $\mathrm{mL}$ distilled water $+1.5 \mathrm{~mL}$ Folin reagent, using 1.2 $\mathrm{mL} \mathrm{Na}_{2} \mathrm{CO}_{3}$ as blank solution, and measured by UV-Vis Spectrophotometer (Hitachi U1800) at a $765 \mathrm{~nm}$ wavelength. Gallic acid was used as the standard curve. Sample results were presented in $\mathrm{mg}$ gallic acid equivalent $(\mathrm{GAE}) / \mathrm{mL}$.

\section{Total Flavonoid Content}

The method is adopted from Mansur et al. ${ }^{18}$. The standard curve determination was carried out using 5, 10, 15, 20, $25,30,35,50 \mathrm{ppm}$ serial dilution of quercetin in Et-OH. 2 grams of aluminum chloride was diluted in $100 \mathrm{~mL}$ Et$\mathrm{OH}$. The experiment is conducted in a fume hood as $\mathrm{AlCl}_{3}$ is corrosive. $2 \mathrm{~mL}$ of $\mathrm{AlCl}_{3}$ and $2 \mathrm{~mL}$ of each quercetin concentration solution were transferred into individual test tubes, incubated, vortexed and measured at $415 \mathrm{~nm}$ using a spectrophotometer. For sample measurement, $2 \mathrm{~mL}$ of the sample ethanolic extract is added to $2 \mathrm{~mL} \mathrm{AlCl}_{3} \quad(2 \%$ solution) in each tube and then measured. The sample is presented in mg quercetin equivalent $(\mathrm{QE}) / \mathrm{mL}$.

\section{Qualitative Phytochemical Screening}

\section{Alkaloids}

Identification of Alkaloids was done via methods described in Farnsworth, N. ${ }^{19}$. and Depkes RI ${ }^{20}$. The sample contains alkaloids if at least two groups of the experimental solutions from the three reagents tested form precipitates. To prepare the test solution, $50 \mathrm{mg}$ of the extract was added to $1 \mathrm{~mL} \mathrm{HCL} 2 \mathrm{~N}$ and $9 \mathrm{~mL}$ water and then it was heated in a water bath for 2 minutes, cooled and filtered to obtain the filtrate.

\section{Bouchardat's Reagent}

The reagent was made of $2 \mathrm{~g}$ iodium $\mathrm{P}$ and $4 \mathrm{~g} \mathrm{Kl} \mathrm{P}$ that were dissolved in $100.0 \mathrm{~mL}$ distilled water. $1 \mathrm{~mL}$ filtrate was added to 2 drops of the Bouchardat Reagent. If a brownblack precipitate is formed, the sample is positive, containing alkaloids.

\section{Mayer's Reagent}

The reagent was made of a mixture of a solution of mercury (II) chloride $\mathrm{P}\left(1.358 \mathrm{~g}\right.$ of $\mathrm{HgCl}_{2}$ in $60 \mathrm{~mL}$ of distilled water) and a solution of potassium iodide $\mathrm{P}$ ( $5 \mathrm{~g}$ of potassium iodide in $10 \mathrm{~mL}$ of distilled water) was combined with distilled water until the volume of the entire mixture reached $100 \mathrm{~mL}$. $1 \mathrm{~mL}$ filtrate was added to 2 drops of the Mayer's Reagent. If a lumpy white or yellow precipitate is formed that dissolves in methanol, the sample is positive, indicating the presence of alkaloids.

\section{Dragendorff's Reagent}

The reagent was made of a mixture of bismuth nitrate $\mathrm{P}$ ( $8 \mathrm{~g}$ of bismuth nitrate in $20 \mathrm{~mL}$ nitric acid) and potassium iodide $\mathrm{P}$ solution $(27.2 \mathrm{~g}$ potassium iodide $\mathrm{P}$ in $50.0 \mathrm{~mL}$ distilled water) which was left to stand until completely separated. The clear solution is taken and dissolved in distilled water until the entire mixture's volume reaches $100.0 \mathrm{~mL}$. $1 \mathrm{~mL}$ of the filtrate was added to 2 drops of the Dragendorff's Reagent. If an orange-brown precipitate is formed, the sample is positive, containing alkaloids.

\section{Flavonoid}

Identification of flavonoid content was done by following the procedure in ${ }^{20}$. A few mg of extract was added to $4 \mathrm{~mL}$ ethanol $\mathrm{P}$ until the extract was dissolved. $2 \mathrm{~mL}$ of the solution was added to 0.5 grams of zinc powder and $2 \mathrm{~mL}$ of $2 \mathrm{~N} \mathrm{HCl}$ and was left to stand for 1 minute. Then 10 
drops of concentrated $\mathrm{HCl} \mathrm{P}$ were added. An intensive red colour observed within 2-5 minutes indicates the presence of flavonoids (glycoside-3-flavonol).

\section{Tannin}

Identification of Tannin was done as illustrated in Farnsworth, Norman R.'s work ${ }^{19}$. A few mg of the thick extract was added to $15 \mathrm{~mL}$ of hot water. Then heated until mixture boils for 5 minutes. Filtrate was filtered. A few drops of $1 \% \mathrm{FeCl}_{3}$ was added to produce a green-violet colour.

\section{Saponin}

$5 \mathrm{mg}$ of the extract was added to a test tube to which 10 $\mathrm{mL}$ of hot water was also added. Mixture was cooled and shaken vigorously for 10 seconds. A steady foam formed 1 to $10 \mathrm{~cm}$ high for no less than 10 minutes. With the addition of 1 drop of $2 \mathrm{~N} \mathrm{HCl}$, the foam did not disappear.

\section{Terpenoid}

A total of $5 \mathrm{mg}$ of the concentrated extract was added to $3 \mathrm{~mL}$ of dichloromethane and then evaporated in an evaporating dish. The evaporated residue is added to 6 drops of acetic acid and 3 drops of concentrated $\mathrm{H}_{2} \mathrm{SO}_{4}$. The sample is positive for terpenoids if it produces a red-green or violet-blue colour.

\section{Results and Discussion}

As has been structured in the preceding portion of the article, results are reported in two parts, that of the assessments of the properties of A bisporus mushrooms and secondly, identification of the compounds responsible for the observed abilities of the A bisporus.

Part 1: Assessment of Antioxidant, SPF and Tyrosinase-Inhibiting Properties

\section{DPPH Radical Scavenging Activity}

The Agaricus bisporus extract's free-radical scavenging capacity was measured by the DPPH (2, 2-diphenyl-1picrylhydrazyl) method. The assay relies on measuring the absorbance of the naturally purple-coloured DPPH with respect to how much the sample can quench it, rendering the solution colourless. The antioxidant compound donates a hydrogen atom to the DPPH radical solution which is then reduced. The absorption of the hydrogen by the antioxidant, namely the A bisporus given that it contains the bioactive compounds for the mushroom to act as an antioxidant, is in stoichiometric ratios with respect to the level of reduction and the DPPH remaining after the radical-scavenging effect. Upon measurement following a set amount of time, the absorption/reduction of the hydrogen is inversely related with the radical scavenging capabilities of the antioxidant ${ }^{21}$. The results were reported as $\mathrm{IC}_{50}$ values, the concentration required to achieve $50 \% \mathrm{RSA}$.

As a baseline for understanding the Agaricus bisporus's ability to scavenge radicals compared to a given standard, a brief review of existing literature will be presented prior to discussion of the results. Abdullah et al.'s ${ }^{21}$, research of selected culinary medical mushroom's antioxidant effects reports positive control (quercetin) DPPH scavenging ability of IC50 $=0.03 \mathrm{mg} / \mathrm{mL}$ whose value can be interpreted as excellent, while the various mushroom extracts exhibited radical scavenging ability of $5.28-35.6 \mathrm{mg} / \mathrm{mL}$ which was suggested still to be viable (although quite low in general) for prospects of the mushroom sample being primary antioxidants whose consumption could reduce the risk of cardiovascular disease and act as a protective agent against oxidative stress.

Another study by Gasecka, et al. ${ }^{7}$ reports an $\mathrm{EC}_{50}$ value for DPPH radical scavenging effective concentration, that ranges from $\sim 800$ to $3200 \mu \mathrm{g} / \mathrm{mL}$ for the A.bisporus species. Amongst the mushroom species investigated in Ga secka, et al.'s study, the white A.bisporus showed the least effective inhibition.

As a comparison of what is considered a highly effective antioxidant, in Table 2, results of Di Petrillo et al.'s ${ }^{22}$ study is shown, elucidating the antioxidant properties of the derived ethanolic extracts of Asphodelus microcarpus medicinal plants, with DPPH scavenging IC50 values of 55.9 \pm 1.55 for the leaves of the plant, $28.4 \pm 0.85$ for its flowers and $360 \pm 56.57$ for its tubers.

In Table 2 and Figure 1, the \%RSA of the A bisporus ethanolic extract is shown. Results conclude that the A bisporus ethanolic extract presents IC50 of $\sim 5456 \mu \mathrm{g} / \mathrm{mL}$. In Table 3, a summary of IC50 values from previously reported works are compiled and used for result comparison. A lower IC50 value is indicative of stronger antioxidant activity, hence, using Abdullah et al.'s ${ }^{21}$ work as a basis for comparison, the ethanolic extract of A.bisporus mushrooms is considered moderately efficient as radical scavenging agents. In Abdullah et al.'s work ${ }^{21}$, a much higher IC50 is reported. However it must be noted that in the mentioned study, the group evaluates the possibility of using hot water for extraction as this would yield the least amount of chemical residues in the product. The high temperatures used in the extraction may cause detrimental effects to the metabolites in the samples, thus leading to a very high IC50 value.

Comparing with the results reported by $\mathrm{Ga}_{\mathrm{s}}$ secka, et, al. ${ }^{7}$, we find that our IC50 value is slightly higher than theirs. This difference may stem from the fact that Gasecka et al. utilizes $70 \%$ ethanol as extraction solvent whereas in our work absolute ethanol is used. The diluted ethanol may be more effective in extracting the metabolites that are responsible for the antioxidant property, in line with similar observations in other studies $^{23}$.

Though Di Petrillo's ${ }^{22}$ research is not of any species of mushrooms, rather a medicinal plant with vastly differing features than the A. bisporus mushrooms, it is of notable significance to note that compared to the range of IC50 values in $\mu \mathrm{g} / \mathrm{mL}$ observed for the plant, from 28.4 in the flowers to 360 in the tubers.

Table 2. Antioxidant activity of Agaricus bisporus. (DPPH) radical scavenging assay.

\begin{tabular}{lll}
\hline Concentration & Abs & \%RSA \\
Control & 1.249 & - \\
10000 & 0.196 & 84.307 \\
8000 & 0.310 & 75.180 \\
6000 & 0.550 & 55.965 \\
4000 & 0.752 & 39.792 \\
2000 & 1.034 & 17.214
\end{tabular}

\section{SPF Test}

A very minimal amount of earlier works have pioneered the research of SPF of specifically the A.bisporus mushroom 
Table 3. Comparison of DPPH radical scavenging ability of Agaricus bisporus ${ }^{7}$ other mushrooms ${ }^{21}$ and Asphodelus microcarpus ethanolic extract ${ }^{22}$

$$
\begin{aligned}
& \text { Asphodelus microcarpus (ethanolic extract, } \\
& \mu \mathrm{g} / \mathrm{mL})^{22}
\end{aligned}
$$

\begin{tabular}{|c|c|c|}
\hline $\begin{array}{l}\text { Various } \\
\text { mushroom } \\
\text { species (hot } \\
\text { water extract, } \\
\mu \mathrm{g} / \mathrm{mL})^{21}\end{array}$ & $\begin{array}{l}\text { Agaricus } \\
\text { bisporus } \\
\text { various strain } \\
\text { ( } 70 \% \text { ethanol } \\
\text { extract, } \\
\mu \mathrm{g} / \mathrm{mL})^{7}\end{array}$ & $\begin{array}{l}\text { Agaricu } \\
\text { bisporu } \\
\text { (ethano } \\
\mu \mathrm{g} / \mathrm{mL} \text { ) } \\
\text { Work] }\end{array}$ \\
\hline$\sim 5280-35659$ & $\sim 800-3200$ & $\sim 5456$ \\
\hline
\end{tabular}

\begin{tabular}{llll} 
& Leaves & Flowers & Tubers \\
DPPH & $\sim 55.9$ & $\sim 28.4$ & $\sim 360$ \\
scavenging & & & \\
IC50 & & & \\
\hline
\end{tabular}

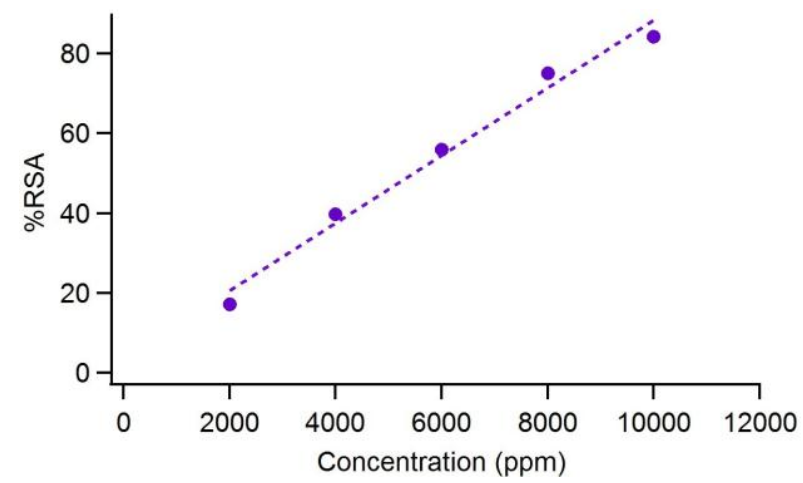

Figure 1. Antioxidant activity of Agaricus bisporus ethanolic extract.

species. Suhaenah et al.'s ${ }^{24}$ work amongst one of the scanty available pieces of literature, reported a range of SPF values at different concentrations, of 17.672 up to 31.325 at higher concentrations, evidencing the claim that the A.bisporus mushroom species is capable to providing medium to the lower end of high SPF, protection against UVR.

Additionally, Hailun et al's ${ }^{25}$ comprehensive review of natural components commonly found in market sunscreens report various compounds such as Silymarin, an essential

compound for topical photoprotection, renowned for its antioxidant properties and potential as a protector against solar radiation, independently having an SPF value of 5.50 \pm 0.25 , at a concentration of $50 \mu \mathrm{mol} / \mathrm{L}$, processed as an ethanolic solution and analysed in vitro. When combined

with chemical sun-protecting agents titanium dioxide and zinc oxide, the observed effect was an increased SPF ability, at $12.37 \pm 4.39$ and $16.30 \pm 2.98$, respectively. Plant-

derived extracts that have been recently incorporated in market sunscreens including Sphaeranthus indicus, rich in phenols and flavonoids were found to have SPF values of 3.85 at $10000 \mathrm{ppm}^{26}$. The renowned cancer-preventing plant Moringa oleifera, rich in polyphenols, some of which include "quercetin, rutin, chlorogenic acid, ellagic acid, and ferulic acid that can be applied in sunscreens" report

SPF values of 2 at concentration of 20000 to 40000 $\mathrm{ppm}^{27}$. Chitosan/tripolyphosphate (TPP) nanoparticles "with vegetable extracts rich in flavonoids provides a topical formulation against sunburns" and were reported to have an $\mathrm{SPF}$ of $2.3 \pm 0.4$ at $200 \mathrm{ppm}$ concentration ${ }^{28}$. Furthermore, the study highlights the synergic effects of these natural ingredients and extracts in market sunscreen formulation with many of the natural sunscreens enhancing the protective effects of chemical sunscreens.

In our work we find that the A.bisporus ethanolic extract with a concentration of 5,000 ppm has SPF value of 5.355 (Table 4). This value is relatively low in comparison to A. bisporus ethanolic extract reported by Suhaenah et al. ${ }^{24}$ Based on their reported data for extract with $1 \%$ to $4 \%$ solution, which equates to 10000 to $40000 \mathrm{ppm}$, by extrapolating the dataset (Figure 2), we can expect an SPF value of $\sim 11$ for their samples at $5000 \mathrm{ppm}$ (Figure 2). This is higher than the SPF value found in our work. In comparison with other compounds, the general standard for low, medium and high SPF values are between 2 to 11,12 to 29, and 30 to 50 , respectively ${ }^{24}$. The higher the concentration, the higher the SPF value in general. In most cases, concentration of extract under test ranges between 0.1 to $10 \%$ or 1000 to $100000 \mathrm{ppm}$.

Although the standard classification for SPF strength given by Suhaenah et al. ${ }^{24}$ and regarded universally with regards to conventional sunscreens would categorize an extract with $5000 \mathrm{ppm}$ concentration having the value 5.355 as being a low SPF agent, when viewed in the context of it being a natural ingredient, this value is relatively favorable. This is bolstered by the similar and even lower values reported in Hailun et al's ${ }^{25}$ study for the various natural compounds reviewed in their study such as the Sphaeranthus indicus, Moringa oleifera, Ginkgo biloba L., and others. Furthermore, it is interesting to note Hailun et al's study's ${ }^{25}$ remarks on the importance of viewing natural agents as enhancers rather than stand-alone UVR protectors. A.bisporus mushrooms may likewise pose as valuable agents to be further studied and utilized in the skin-care and medical industry with regards to the excessive melanogenesis discussed earlier.

\section{Tyrosinase Inhibition Test}

Tyrosinase, as aforementioned, is an oxidase comprised of copper and an enzyme that catalyses the synthesis of melanin. The rate-limiting enzyme has the role of converting tyrosine into dihydrophenylalanine (DOPA) and further oxidize this DOPA into dopaquinone. Melanin inherently is a natural mechanism of the human body however the over-secretion of melanin from melanocytes is what comprises the main health issue, caused by external factors such as exposure to UVR and release of $\alpha$ melanocyte-stimulating hormones ${ }^{29}$. The overexpression of tyrosinase therefore leads to the overproduction of melanin associated with hyperpigmentation, freckles or 
Table 4. SPF of Agaricus bisporus ethanolic extract at $5000 \mathrm{ppm}$ concentration . Raw data calculations from absorbance at wavelengths 290-320, sample diluted with dilution factor 3 .

\begin{tabular}{|c|c|c|c|c|c|c|c|c|}
\hline & Abs & Dilution factor & Abs Final & EE $\times$ I & Abs x EE x I & Total & Correction factor & SPF \\
\hline 290 & 0.46 & 3 & 1.386 & 0.0150 & 0.02 & & & \\
\hline 295 & 0.43 & 3 & 1.293 & 0.0817 & 0.11 & & & \\
\hline 300 & 0.23 & 3 & 0.678 & 0.2874 & 0.19 & & & \\
\hline 305 & 0.13 & 3 & 0.390 & 0.3278 & 0.13 & 0.536 & 10 & 5.355 \\
\hline 310 & 0.10 & 3 & 0.312 & 0.1864 & 0.06 & & & \\
\hline 315 & 0.09 & 3 & 0.282 & 0.0839 & 0.02 & & & \\
\hline 320 & 0.09 & 3 & 0.255 & 0.0180 & 0.00 & & & \\
\hline
\end{tabular}

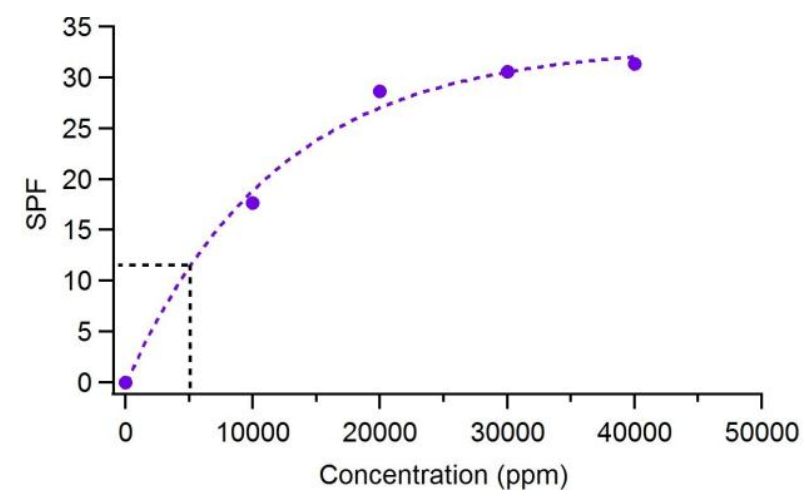

Figure 2. Extrapolation of SPF vs concentration data reproduced from ${ }^{24}$ regarding ethanolic extract of Agaricus bisporus. Based on this extrapolated data, SPF of $\sim 11$ is expected for $5000 \mathrm{ppm}$ in the samples of Suhaenah et al. ${ }^{24}$

melanoma. Hence, tyrosinase inhibitors have attracted much attention by various industries, not only the cosmetic industry but also in medicine and pharmacology. Kojic acid, a synthetic tyrosinase inhibitor widely utilized in skinwhitening agents, is used as a positive control in the current study. However, these synthetic compounds have been evidenced to be associated with side effects such as erythema or contact dermatitis, increasing interest towards natural and biocompatible agents ${ }^{30}$, sparking the interest of determining the tyrosinase inhibiting activities of A.bisporus mushrooms, whose properties with regards to tyrosinase inhibition is minimally documented in literature.

As a baseline for evaluation, Di Petrillo et al.'s ${ }^{22}$ results are tabulated in Table 5 showing tyrosinase inhibiting ability of Asphodelus microcarpus medicinal plants with an IC50 value of $270 \mu \mathrm{g} / \mathrm{mL}$. Furthermore, the tyrosinase inhibiting properties of A.bisporus and other mushrooms studied by Taofiq et al. ${ }^{29}$ are also listed for comparison. They 29 found that A.bisporus ethanolic extracts had anti-tyrosinase potential with an EC50 value in $\mathrm{mg} / \mathrm{mL}$ of $160 \mu \mathrm{g} / \mathrm{mL}$, the highest among the studied mushroom species which included P.ostreatus and L.edodes. In addition, the literature cited in Taofiq et al.'s report, namely Miyake et al.'s ${ }^{31}$ confirms that the phenolic compound 2-amino-3H-phenoxazin-2-one, isolated from A.bisporus, at $0.5 \mu \mathrm{M}$, could significantly inhibit the enzyme tyrosine up to $80 \%$. To further reinforce this, Yan et al. 's study ${ }^{32}$ describes that the isolated phenolic acids: steroidal triterpenes, namely botulin and trametenolic acid, found in mushroom extracts inhibit tyrosinase activity with an IC50 of $5.13 \mu \mathrm{M}$, stronger than the IC50 value of their positive control kojic acid at $6.43 \mu \mathrm{M}$, and 7.25 $\mu \mathrm{M}$ respectively. These results suggest that the constituent bioactive phenolic compounds of mushrooms, including A.bisporus can be exploited as tyrosinase-inhibiting agents. Their work is consistent with that of Di Petrillo et al ${ }^{22}$ on the phenolic rich Asphodelus microcarpus which is shown to exhibit high antioxidant and tyrosinase inhibition properties.

In our work, we find that the Agaricus bisporus ethanolic extract exhibits an exceptionally high tyrosinase inhibiting activity. As compared to kojic acid as the positive control, the Agaricus bisporus ethanolic extract shows significantly higher inhibition of tyrosinase (Figure 3). IC50 value of kojic acid was $135.50 \mathrm{ppm}$ or $\mu \mathrm{g} / \mathrm{mL}$. On the other hand, the extract sample shows strong activity at very small concentration. Almost complete inhibition is observed for samples with concentration larger than $3 \mu \mathrm{g} / \mathrm{mL}$. Between $0.01 \mu \mathrm{g} / \mathrm{mL}$ and $3 \mu \mathrm{g} / \mathrm{mL}$, we observed a gradual increase in the tyrosinase inhibition. Figure $3 a$ shows the data for three separate trials, showing similar trends. It was quite challenging in obtaining precise IC50 for the extract due to the very low concentration of extract. From Figure 3.a, IC50 for the Agaricus bisporus ethanolic extract is estimated to be $\sim 2 \mu \mathrm{g} / \mathrm{mL}$. Our result shows stronger tyrosinase inhibition capability than that reported by Taofiq et al. ${ }^{29}$. In general, our result is consistent in that there is a substantial tyrosinase inhibiting properties in the A.bisporus mushroom as reported by Taofiq et al. ${ }^{29}$, Miyake et al. ${ }^{31}$ and Yan et al. ${ }^{32}$. The A.bisporus ethanolic extract in our work is also shown to have better tyrosinase inihibiting performance compared to that by Asphoedulus microcarpus in Table $5^{22}$, further supporting the conclusion that A.bisporus mushrooms are superior tyrosinase inhibitors and thus hyperpigmentation or other melanin-related disorder correctors.

The difference between our values with that of Taofiq et $\mathrm{al}^{29}$ might be associated with the slightly different extraction methods. In their work, samples are first dried at $30^{\circ} \mathrm{C}$ in an oven and processed into fine powder, followed by 12 cycles of reflux for 4 hours in an assumed $78{ }^{\circ} \mathrm{C}$ (ethanol reflux temperature), and finally evaporated under reduced pressure using a rotary evaporator to obtain the dried ethanolic extract. In our work, relatively milder sample processing conditions are used which may slightly improve the preservation of the metabolites in the sample. Samples are first freeze fried for 72 hours and ground into fine powder. Maceration was done at room temperature for 24 hours followed by filtration and solvent evaporation under reduced pressure in a rotary evaporator.

Part 2: Identification of Phenolic, Flavonoid, and Other Phytochemicals 

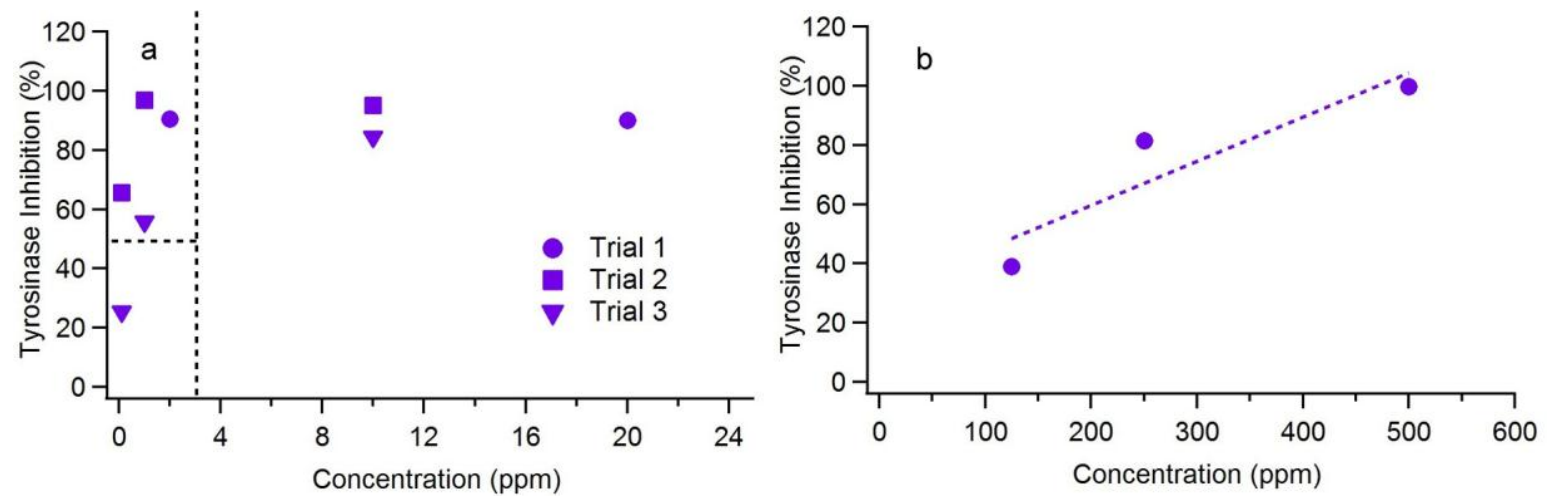

Figure 3. Tyrosinase inhibition of (a) Agaricus bisporus ethanolic extract in this work in comparison to the (b) kojic acid control samples. Dotted lines in (a) are guiding lines.

Table 5. Comparison of tyrosinase Inhibition of Agaricus bisporus in this work against those in ${ }^{29}$ and Asphodelus microcarpus ethanolic extract reported in ${ }^{22}$

\begin{tabular}{|c|c|c|c|c|c|c|c|c|}
\hline & \multicolumn{3}{|c|}{ Asphodelus microcarpus $(\mu \mathrm{g} / \mathrm{mL})^{22}$} & \multirow{2}{*}{$\begin{array}{l}\text { Lentinula } \\
\text { edodes } \\
(\mu \mathrm{g} / \mathrm{mL})^{29}\end{array}$} & \multirow{2}{*}{$\begin{array}{l}\text { Postreatus } \\
\text { ostreatus } \\
(\mu \mathrm{g} / \mathrm{mL})^{29}\end{array}$} & \multirow{2}{*}{$\begin{array}{l}\text { Agaricus } \\
\text { bisporus } \\
(\mu \mathrm{g} / \mathrm{mL})^{29}\end{array}$} & Agaricus & \\
\hline Tyrosinase & Leaves & $\begin{array}{c}\text { Flowers } \\
\sim 270\end{array}$ & Tubers & & & & $\sim 2$ & \\
\hline $\begin{array}{l}\text { Inhibition } \\
\text { IC50 }\end{array}$ & & & & & & & & \\
\hline
\end{tabular}

To gain insight on the possible origin of the observed properties of the Agaricus bisporus ethanolic extract, next we report selected phytochemical analysis that we carry out. Total phenolic content analysis is carried out quantitatively while the flavonoid content analysis is carried out both quantitatively and qualitatively. Other secondary metabolites are screened qualitatively.

\section{Total Phenolic Content by Folin Reagant Method}

Phenolic compounds are commonly found in many plants, namely also mushrooms and are indicative of and closely related with antioxidant activities.

Ga secka, et, al. ${ }^{7}$ studied the profile of phenolic, organic acids and antioxidant properties of various species of Agaricus mushrooms. Their report demonstrated that various strains of white A. bisporus mushroom species has a total phenolic content in the range of 132-756 mg GAE/100g DW.

Furthermore, Alispahic' et al.'s ${ }^{33}$ study of the phenolic content of various species of mushrooms (dry boletus, white and brown champignon, oyster and shiitake mushrooms) showed total phenolic content of 494-3556 mg GAE/100gand concluded that these mushroom species were potential sources of natural antioxidants. The highest total phenolic content was observed in dry boletus mushrooms.

In this work, we determine the total phenolic content in Agaricus bisporus ethanolic extracts to be $\sim 1143 \mathrm{mg}$ GAE/100g (Figure 4 and Table 6). This value exceeds that of $\mathrm{Ga}_{c}$ secka, et, al.'s ${ }^{7}$ and lies within the range of values reported by Alispahic' et al ${ }^{33}$. The different total phenolic content compared to previously reported work could be due to different extraction methods.

Total Flavonoid Content

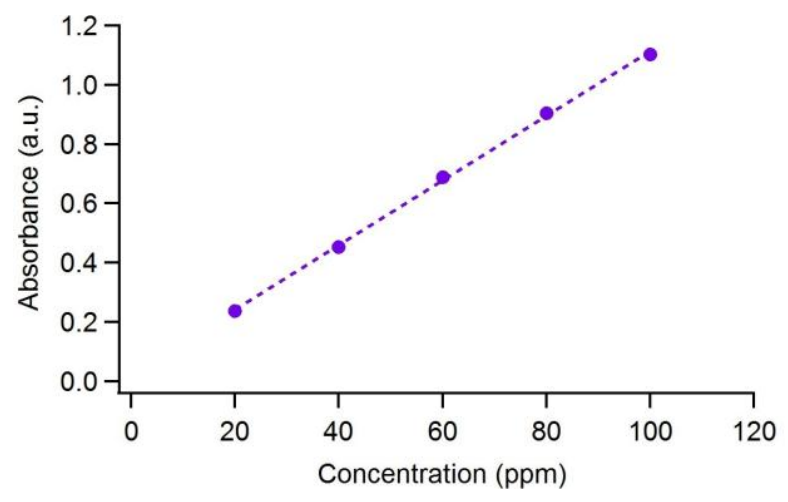

Figure 4. Calibration curve of gallic acid standard for determination of total phenolic content of Agaricus bisporus ethanolic extract.

Flavonoids are a group of natural substances with variable phenolic ${ }^{34}$, generally found in vascular plants as glycosides and flavonoid aglycones. Typically in analysing flavonoids, the aglycones in the plant extracts that have been hydrolysed are examined. The flavonoid extraction is carried out with boiled ethanol to avoid enzyme oxidation ${ }^{35}$. Flavonoids are renowned for a broad spectrum of favourable healthpromoting benefits and hence are considered indispensable components in diverse applications in the nutraceutical, pharmaceutical, medicinal and cosmetic industry. This can be attributed to their "anti-oxidative, anti-inflammatory, anti-mutagenic and anti-carcinogenic properties coupled with their capacity to modulate key cellular enzyme function" with an added impulse for lowering cardiovascular mortality rate and association with diseases such as cancer, Alzheimer's disease and atherosclerosis among others ${ }^{34}$. 
Table 6. Total phenolic content of Agaricus bisporus ethanolic extract measurement and calculation.

\begin{tabular}{lllcc} 
& Abs. & Conc. (mg/L) & Unit Conv. [x 1L/5g (mg GAE/g)] & Ave. (mg GAE/100g) \\
Sample 1 & 0.562 & 49.33945 & 9.878 & 1143.2 \\
Sample 2 & 0.732 & 64.93578 & 12.987 & \\
\hline
\end{tabular}

Gil-Ramírez et al.'s ${ }^{36}$ study which testifies to the claim that mushrooms do not contain flavonoids and cannot actually synthesize flavonoids as they lack the required enzymes. The general trend in scientific literature testifying to the presence of flavonoids in mushrooms is critiqued for their uses of unspecific colorimetric methods developed originally to quantify flavonoids in plants as a general category. Furthermore, sequences of DNA encoding for the key enzymes involved in the flavonoid biosynthetic pathway were not discovered in the genomic databases of completely sequenced mushrooms. It was evidenced that the fruiting bodies of mushrooms were also incapable of absorbing flavonoids from their surroundings. These results also agree with that of $\mathrm{Ga}_{c}$ secka, et, al.'s ${ }^{7}$ study that reports that flavonoids were not found in all the mushroom species experimented, including white Agaricus bisporus.

The determination of the total flavonoid content in Agaricus bisporus was carried out with ethanol extracted samples using quercetin as standard (Figure 5 and Table 7). Total flavonoid content of A.bisporus mushrooms is 0.759 $\mathrm{mgQE} / \mathrm{g}$, a very minimal quantity. In retrospect with past studies, these results agree with that of Gil-Ramírez et al.'s ${ }^{36}$ and $\mathrm{Ga}_{c}$ secka, et al.'s ${ }^{7}$, confirming the absence or very minor amount of flavonoids in Agaricus bisporus mushrooms. The lack of flavonoid in our sample is also confirmed by the qualitative phytochemical screening shown in Figure 6.

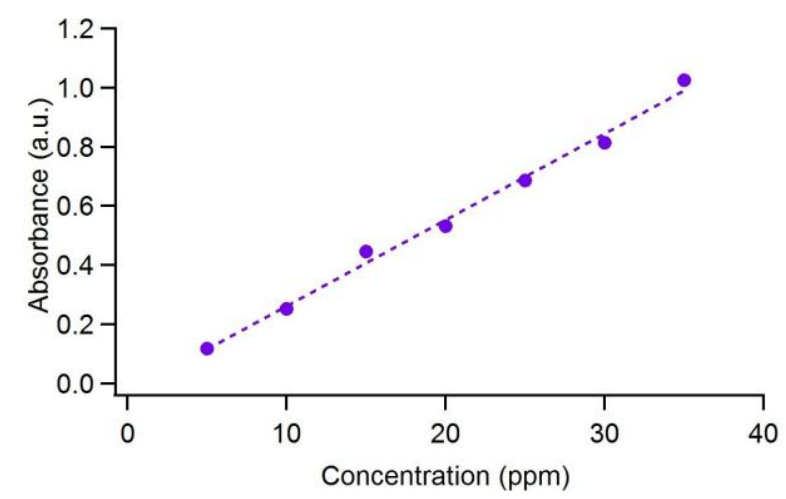

Figure 5. Calibration curve of quercetin standard for determination of total phenolic content of Agaricus bisporus ethanolic extract.

\section{Qualitative Phytochemical Screening}

The phytochemical screening is a qualitative test of the chemical contents of plants in order to determine the class of compounds contained in the extracts derived from plants. The screening analyses the presence of secondary metabolites like alkaloids, flavonoids, terpenes, tannins, saponins, terpenoids, glycosides, quinones and anthraquinones in the extracts ${ }^{35}$.

Several works reported phytochemical screening of Agaricus bisporus. Eswari et al. ${ }^{37}$ studied A. bisporus petroleum ether and methanolic extracts and found that alkaloid is only detected in the petroleum ether based extracts. Flavonoid was weakly detected in the methanolic extract and not in the petroleum ether extract. Tannin was strongly detected in petroleum ether extracts and not in the methanolic extract. Saponins are strongly detected in methanolic extract and not in petroleum ether extract. Terpenoid is weakly and moderately detected in the petroleum ether and methanolic extracts, respectively.

In our works, the extraction was carried out with absolute ethanol. The ethanolic extract in our work shows only the presence of terpenoid (Figure 6). This result generally agrees with the work of Eswari et al ${ }^{37}$ except for the lack of detection for saponin. The presence of terpenoid indicates that $\mathrm{A}$. bisporus has another potential application in the field of therapeutics medicine. Indeed, there appears to be renewed interest in the study of mushrooms as a natural source of terpenoids ${ }^{38}$. Several groups have reported the efficacy of terpenoids for therapeutic treatments of neurodegenerative ${ }^{39}$, antiviral ${ }^{40}$, and more. Several studies have looked at more closely the health benefits of terpenoid extracted from various mushrooms such as Ganodherma ludicum ${ }^{41}$, Antrodia cinnamomea ${ }^{42}$, Inonotus obliquus ${ }^{43}$, and others, but to the author's knowledge, there has not been a study of therapeutics effects of terpenoid extracts from Agaricus bisporus. The presence of terpenoid could also be one of the high tyrosinase inhibition activity in the Agaricus bisporus in our work. Both phenolic and terpenoid compounds have been found to directly contribute to tyrosinase inhibiting activities ${ }^{44}$.

Several things are interesting to note. First, our work agrees with that of others in that there is an absence or very minimal amount of flavonoid in the Agaricus bisporus mushrooms. Our work supports the prior theory and analysis which suggest the inability of the mushroom to produce flavonoids due to the lack of required enzyme and/or that the mushroom fruiting body is incapable of absorbing flavonoids from the surrounding.

Secondly, our work agrees with the prior reports ${ }^{7}, 21$ that demonstrated Agaricus bisporus as the least effective radical scavenging agents compared to other common edible mushrooms. It is interesting also to note that our sample exhibits a higher amount of total phenolic content compared to that of Agaricus bisporus in other works ${ }^{7},{ }^{21}$ but we observed a lower radical scavenging capability in our sample than expected. Thus, it can be said that the phenolic compound in Agaricus bisporus plays a minor role in radical scavenging.

Likewise, a lower value of SPF is also found compared to that of other works ${ }^{25}$ but remains favorable compared to some plant sources ${ }^{24}$. It is unclear whether the total phenolic content in our Agaricus bisporus is higher or lower than that in ${ }^{25}$ since it was not reported.

The high amount of total phenolic content in our sample does however correlate with the exceptional tyrosinase inhibition capability we are seeing in our Agaricus bisporus sample. Such correlation between phenolic and tyrosinase 
Table 7. Total flavonoid content of Agaricus bisporus ethanolic extract measurement and calculation.

\begin{tabular}{lllcc}
\hline & Abs. & Conc. (mg/L) & Unit Conv. [x 1L/10g (mg QE/g)] & Ave. (mg QE/g) \\
Sample 1 & 0.206 & 8.072 & 0.807 & 0.759 \\
Sample 2 & 0.178 & 7.113 & 0.711 & \\
\hline
\end{tabular}

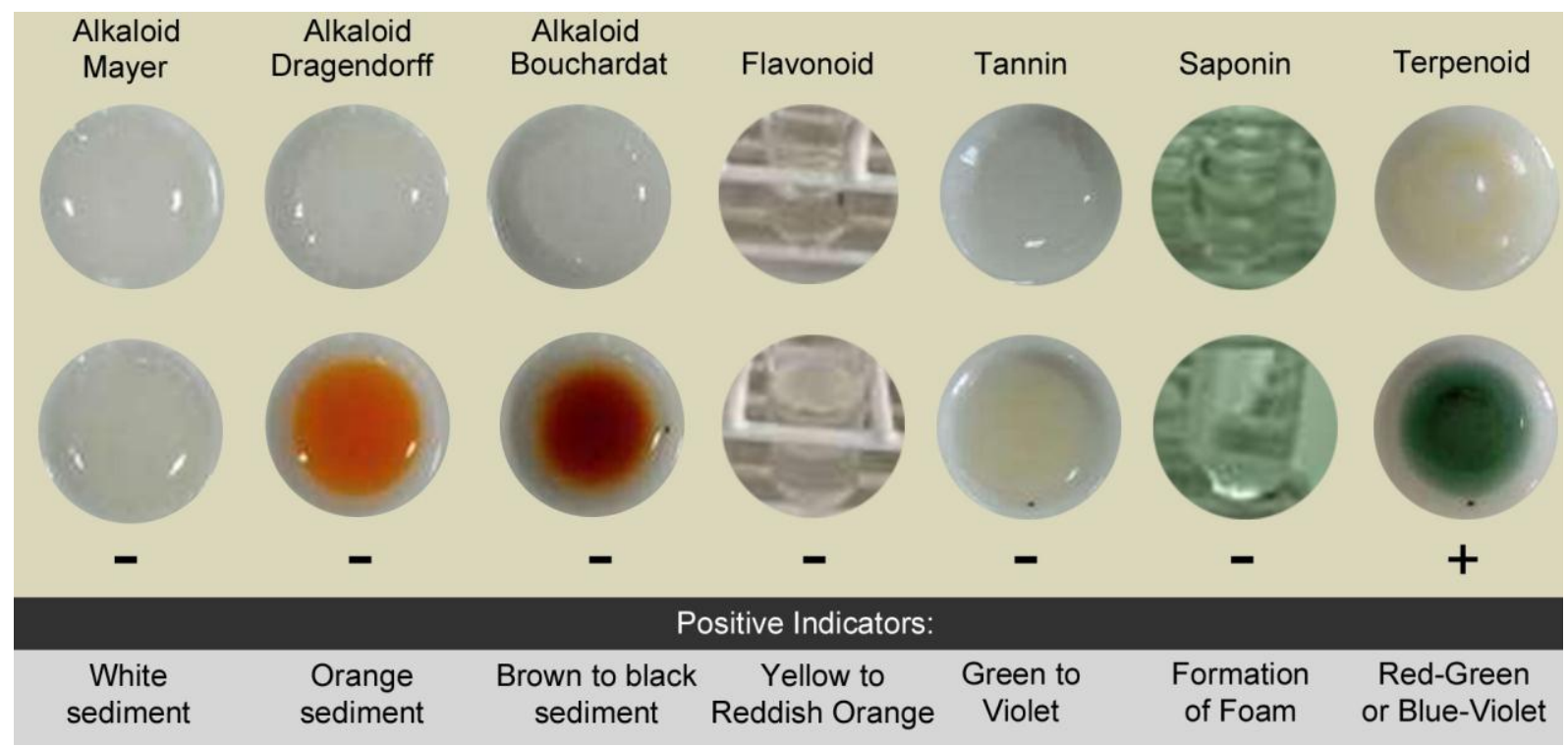

Figure 6. Qualitative phytochemical testing observation. Only terpenoid is clearly detected in the Agaricus bisporus ethanolic extract.

inhibition have been seen in many systems ${ }^{38}$. The correlation is further bolstered by the fact that our qualitative phytochemical screening established no alkaloid contents in the Agaricus bisporus ethanolic extract we examine. It is known that alkaloid is one of the drivers for tyrosinase inhibition activity ${ }^{38}$.

Besides alkaloid, terpenoid is also known to be one of the drivers for tyrosinase inhibition activity ${ }^{38}$ and indeed the terpenoid is present in our sample. It is not known how much is present in the sample but it is plausible that terpenoid in the sample contributes to both the radical scavenging and tyrosinase inhibiting properties we observe in our sample.

Thus, from the aforementioned results and discussion, it is observed that the strong tyrosinase inhibition in the Agaricus bisporus ethanolic extract is likely to be driven by the phenolics and terpenoids in the sample. More work however is required to elucidate further the types and distribution of phenolics and terpenoids in the sample among other more quantitative phytochemical analysis. We note that in our work we have minimized the use of higher temperature processings during the extraction and that we are using absolute ethanol as solvents instead of the more commonly used methanol and diluted ethanol. This combination of processing may result in different metabolites being extracted, which gives rise to some different behavior compared to other Agaricus bisporus work.

\section{Conclusion}

In conclusion, our work agrees with previously reported work with some variations. We find that indeed the common button mushroom namely the Agaricus bisporus is available as a minor source of antioxidant agent with \%RSA IC50 of $\sim 5456 \mu \mathrm{g} / \mathrm{mL}$. It also presents as a moderate source of sun protecting agent with SPF value of $\sim 5.355$ at $5000 \mathrm{ppm}$ concentration, prior to combination and supplementation with chemical UV filters such as titanium oxide or zinc. More work is required to further evaluate the SPF in our sample. It would be especially useful to obtain more SPF value at higher concentrations in order to compare its efficacy with those in other works. Of note, we observed a high tyrosinase inhibiting capability of the Agaricus bisporus ethanolic extract with IC50 of $\sim 2 \mu \mathrm{g} / \mathrm{mL}$. This is seen to be likely attributable to the phenolic contained in the mushrooms, found to have a value of $\sim 1143 \mathrm{mg}$ GAE/100g and possibly partially attributable to the terpenoid present in our sample. Additionally, this work highlights the complex and sensitive biochemistry present in the seemingly common mushroom where it is possible that slight differences in extraction method, geographical conditions and cultivation would result in different properties of the extracted products. Overall, through this assessment, it is clear that Agaricus bisporus presents a really exciting outlook for the cosmetic industry and the field of pharmaceuticals where the search for natural compounds over synthetic products has been burgeoning with the rising concerns and prevalent health-issues related with UV radiation and exposure to free-radicals being the cause of oxidative stress.

\section{Acknowledgement}

We are grateful for the funding and support of Sekolah Pelita Harapan Lippo Village through its Applied Science Academy program. The authors are thankful for the generous support and access to the research facility at Universitas 
Pelita Harapan and Emmerich Research Center. C.Y.H., Y.H., M.S., D.R. designed the experiment and carried out the extraction, purification, and characterization of the materials. C.Y.H., Y.H., S.P.W., E.S. carried out the analysis and wrote the manuscript.

\section{References}

1. Dunbar B, 2010. URL www.nasa.gov/topics/solarsystem/ features/uv-exposure.html.

2. Boonsong S, Klaypradit W and Wilaipun P, 2016. URL https: //doi.org/10.1016/j.anres.2015.07.002.

3. Dale B, Moon S and Armstrong F. Comprehensive review of ultraviolet radiation and the current status on sunscreens. The Journal of clinical and aesthetic dermatology 2012; 5(9): 1823.

4. Zaidi K, Ali S and Ali A. Effect of Purified Mushroom Tyrosinase on Melanin Content And Melanogenic Protein Expression. Biotechnology Research International 2016; .

5. Chang TS, 2012. URL https://doi.org/10.3390/ma5091661.

6. Brenner $\mathrm{M}$ and Hearing VJ. The protective role of melanin against UV damage in human skin. Photochemistry and photobiology 2008; 84(3): 539-549.

7. Ga secka M, Magdziak Z and Siwulski M. Profile of phenolic and organic acids, antioxidant properties and ergosterol content in cultivated and wild growing species of Agaricus. European Food Research and Technology 2017; 244: 259-268.

8. Wu L, Chen C, Cheng $\mathrm{C}$ et al. Evaluation of Tyrosinase Inhibitory, Antioxidant, Antimicrobial, and Antiaging Activities of Magnolia officinalis Extracts after Aspergillusniger Fermentation. BioMed Research International 2018; .

9. Noreen H, Semmar N, Farman M et al. Measurement of total Phenolic content and antioxidant activity of aerial parts of medicinal plant Coronopus didymus. Asian Pacific Journal of Tropical Medicine 2017; 10(8): 792-801.

10. Cheung L, Cheung P and Ooi V. Antioxidant activity and total phenolics of edible mushroom extracts. Food Chemistry 2003; 81(2): 419-422.

11. Bhushan A and Kulshreshtha M. The Medicinal Mushroom Agaricus bisporus: Review of Phytopharmacology and Potential Role in the Treatment of Various Diseases. Journal of Nature and Science of Medicine 2018; 1(1):4-9.

12. Lamien-Meda A, Lamien C, Compaoré M et al. Polyphenol Content and Antioxidant Activity of Fourteen Wild Edible Fruits from Burkina Faso. Molecules 2008; 13(3): 581-594.

13. Kumara P, K, Kumar S et al. Determination of DPPH Free Radical Scavenging Activity by RP-HPLC, Rapid Sensitive Method for the Screening of Berry Fruit Juice Freeze Dried Extract. Natural Products Chemistry \& Research 2018; 6(5).

14. Romulo A, Zuhud E, Rondevaldova J et al. Screening of invitro antimicrobial activity of plants used in traditional Indonesian medicine. Pharmaceutical Biology 2018; 56(1): 287-293.

15. Chan E, Lim Y, Wong L et al. Antioxidant and Tyrosinase Inhibition Properties of Leaves and Rhizomes of Ginger Species. Food Chemistry 2008; 109(3):477-483.

16. Burger P, Landreau A, Azoulay S et al. Skin Whitening Cosmetics: Feedback and Challenges in the Development of. Natural Skin Lighteners Cosmetics 2016; 3(4).

17. Javanmardi J, Stushnoff C, Locke E et al. Antioxidant Activity and Total Phenolic Content of Iranian Ocimum Accessions. Food Chemistry 2003; 83(4): 151-152.
18. Mansur J, Breder M, Mansur M et al. Determination of Sun Protection Factor by Spectrophotometry. The Journal Brazilian Annals of Dermatology 1986; 61: 121-124.

19. Farnsworth N. Biological and Phytochemical Screening of Plants. Journal of Pharmaceutical Sciences 1966; 55(3): 225276.

20. Depkes RI, 1995.

21. Abdullah $\mathrm{N}$, Ismail $\mathrm{S}$, Aminudin $\mathrm{N}$ et al., 2012. URL https: //doi.org/10.1155/2012/464238.

22. Petrillo AD, González-Paramás AM, Era B et al. Tyrosinase inhibition and antioxidant properties of Asphodelus microcarpus extracts. BMC Complementary and Alternative Medicine 2016; 16(453).

23. Ngo TV, Scarlett CJ, Bowyer MC et al. Impact of Different Extraction Solvents on Bioactive Compounds and Antioxidant Capacity from the Root of Salacia chinensis L. Journal of Food Quality; 2017.

24. Suhaenah A, Tahir M and Nasra. Penentuan nilai spf (sun protecting factor) ekstrak etanol jamur kancing (Agaricus bisporus) secara in vitro dengan metode spektrofotometri uvvis. As-Syifaa Pharmacy Journal 2019; 11(1): 82-87.

25. Hailun H, Anqi L, Shiqin L et al. Natural Components in Sunscreens: Topical Formulations with Sun Protection Factor (SPF). Biomedicine \& Pharmacotherapy 2020; 134: 111-161.

26. Álvarez Gómez F. UV photoprotection, cytotoxicity and immunology capacity of red algae extracts. Molecules 2019; (2): 24-24.

27. Shanuja SK. Exploring the UVB-protective efficacy of melanin precursor extracted from marine imperfect fungus: featuring characterization and application studies under in vitro conditions. Int Microbiol 2018; 21(1-2): 59-71.

28. Bikiaris ND. Innovative skin product emulsions with enhanced antioxidant, antimicrobial and UV protection properties containing nanoparticles of pure and modified chitosan with encapsulated fresh pomegranate juice. Polymers (Basel) 2020; (7): 12-12.

29. Taofiq O, Heleno SA, Calhelha RC et al. Development of Mushroom-Based Cosmeceutical Formulations with AntiInflammatory, Anti-Tyrosinase, Antioxidant, and Antibacterial Properties. Molecules MDPI Basel 1372; : 21-21.

30. Cui H, Duan F, Jia $S$ et al. Antioxidant and Tyrosinase Inhibitory Activities of Seed Oils from Torreya Grandis Fort. Ex Lindl. Biomed Research International 2018; : 1-10.

31. Miyake M, Yamamoto S, Sano O et al. Inhibitory effects of 2amino-3H-phenoxazin-3one on the melanogenesis of murine B16 melanoma cell line. Biosci Biotechnol Biochem 2010; 74 : 753-758.

32. Yan ZF, Yang Y, Tian FH et al. Inhibitory and acceleratory effects of Inonotus obliquus on tyrosinase activity and melanin formation in B16 melanoma cells. Evid Based Complement Altern Med 2014; .

33. Alispahić A, Šapčanin A, Salihović M et al., 2015.

34. Panche A, Diwan A and Chandra S. Flavonoids: an overview. Journal of Nutritional Science 2016; 5(47).

35. Harborne JB. Metode fitokimia, edisi ke dua, itb.

36. Gil-Ramírez A, Pavo-Caballero C, Baeza E et al. Mushrooms Do Not Contain Flavonoids. Journal of Functional Foods 2016; 25: 1-13.

37. Eswari S, Bhavan PS, Kalpana R et al. Phytochemical Characterization of the mushroom Agaricus bisporus and assessment of its nutritional ability in the place of fishmeal for 
survival and growth of the freshwater prawn Macrobrachium rosenbergii post-larvae. Integrative Food. Nutrition and Metabolism 2019; 6: 1-11.

38. Dasgupta A and Acharya K, 2019.

39. Yoo KY and Park SY. Review: Terpenoids as potential antiAlzheimer's disease therapeutics. Molecules 2012; 17: 35243562.

40. Lin LT, Chung CY, Hsu WC et al. Saikosaponin b2 is a naturally occurring terpenoid that efficiently inhibits hepatitis C virus entry. Journal of Hepatology 2015; 62: 541-549.

41. Komoda Y, Shimizu M and Sonoda Y. Ganoderic acid and its derivatives as cholesterol synthesis inhibitors. Chem Pharm Bull 1989; 37: 531-533.

42. Zhang BB, Guan YY and Hu PF. Production of bioactive metabolites by submerged fermentation of the medicinal mushroom Antrodia cinnamomea: recent advances and future development. Crit Rev Biotech 2019; 39: 541-554.

43. Ying YM, Zhang LY and Zhang X. Terpenoids with alphaglucosidase inhibitory activity from the submerged culture of Inonotus obliquus. Phytochem 2014; 108: 171-176.

44. Zolghadri S, Bahrami A, Khan MTH et al. A comprehensive review on tyrosinase inhibitors. J Enzyme Inhib Med Chem 2019; 34: 279-309. 